\title{
Oclusión intestinal por doble invaginación ileo-ileal posoperatoria en una niña de 11 meses de edad
}

\section{Postsurgical intestinal occlusion ileum-ileum double intussusception in eleven-month girl}

\author{
Alfonso Galván-Montaño ${ }^{1}$, Sonia Guzmán-Martínez ${ }^{1}$, Francisco Serrano-Andrade² y \\ Silvia García-Moreno ${ }^{3 *}$ \\ ${ }^{1}$ Servicio de Cirugía Pediátrica; ${ }^{2}$ Servicio de Cirugía General; ${ }^{3}$ Departamento de Investigación. Hospital General Dr. Manuel Gea González, Ciudad \\ de México, México
}

\section{Resumen}

Antecedentes: La invaginación posoperatoria es una complicación inusual en niños. Su incidencia es del 0.01-0.25\%. Caso clínico: Niña de 11 meses a quien se realizó colostomía y 48 horas después presentó dolor abdominal, vómito gastrobiliar y distensión abdominal. Se realizó laparotomía y se encontró una doble invaginación íleo-ileal a $60 \mathrm{~cm}$ de la válvula ileocecal. Discusión: Las invaginaciones intestinales posoperatorias son extremadamente raras y la mayoría se presentan dentro de los primeros 7 días. La etiología de la invaginación posoperatoria es desconocida. Conclusiones: En niños operados con oclusión intestinal debe sospecharse invaginación intestinal y realizar una laparotomía inmediata.

Palabras Clave: Invaginación posoperatoria. Niños. Oclusión intestinal.

\begin{abstract}
Background: Postoperative intussusception is an unusual complication in children; the incidence is $0.01-0.25 \%$. Clinical Case: Female of 11 months who underwent colostomy, $48 \mathrm{~h}$ later presents abdominal pain, vomiting gastrobiliar and abdominal distension. A laparotomy was performed, finding a double ileo-ileal invagination $60 \mathrm{~cm}$ from the ileocecal valve. Discussion: The postoperative intestinal invaginations are extremely rare and most of them occur within the first 7 days. The etiology of postoperative intussusception is unknown. Conclusions: Postoperative children with intestinal occlusion, intussusception should be suspected and an immediate laparotomy should be performed.
\end{abstract}

Key Words: Postsurgical intussusception. Children. Intestinal occlusion.

\footnotetext{
Correspondencia:

*Silvia García-Moreno

Avda. Calzada de Tlalpan, 4800

Col. Sección XVI, Del. Tlalpan

Fecha de recepción: 02-08-2018

Cir Cir. 2020;88(1):88-90 C.P. 14080 , Ciudad de México, México Fecha de aceptación:19-08-2019 E-mail: chivis-1901@ hotmail.com DOI: $10.24875 /$ CIRU.19000642

Contents available at PubMed www.cirugiaycirujanos.com 0009-7411/@ 2019 Academia Mexicana de Cirugía. Publicado por Permanyer. Éste es un artículo open access bajo la licencia CC BY-NC-ND (http://creativecommons.org/licenses/by-nc-nd/4.0/).
} 


\section{Introducción}

La invaginación posoperatoria es una complicación inusual en niños que se sometieron a varios tipos de cirugía. El reconocimiento temprano es difícil por su rareza y sus presentaciones atípicas ${ }^{1-3}$. La incidencia de invaginación posoperatoria es del $0.01-0.25 \%$ en los niños después de la laparotomía y representa el $5-10 \%$ de las obstrucciones intestinales posoperatorias. Además de la baja incidencia, la ausencia de la tríada típica de intususcepción (dolor cólico abdominal, masa abdominal y heces con sangre) y la presencia de íleo posoperatorio dificultan el reconocimiento precoz de la invaginación posoperatoria ${ }^{1-3}$.

Presentamos el caso de una niña de 11 meses que presentó oclusión intestinal por doble invaginación íleo-ileal 48 horas después de habérsele realizado una colostomía.

\section{Caso clínico}

Niña con diagnóstico de ano imperforado y fístula rectovestibular, inicialmente manejada con dilataciones de la fístula. A los 11 meses de edad se le realizó una colostomía de bocas separadas a nivel del sigmoides. Debido a un prolapso de la boca proximal de la colostomía fue reintervenida quirúrgicamente al día siguiente para corrección del prolapso. A las 24 horas la niña presenta dolor abdominal, vómito de contenido gastrobiliar y distensión abdominal con aumento del perímetro de $4 \mathrm{~cm}$ y ausencia de evacuaciones. Se toma radiografía simple de abdomen en la que se observa una importante dilatación de asas de intestino delgado que sugiere oclusión intestinal (Fig. 1). La biometría hemática reportó leucocitos de 12,000 y proteína C reactiva de $6.510 \mathrm{mg} / \mathrm{dl}$. Se decide realizar laparotomía exploradora con el diagnóstico de oclusión intestinal de origen por determinar. Se encontró como causa una doble invaginación íleo-ileal a $60 \mathrm{~cm}$ de la válvula ileocecal, por lo que se realizó desinvaginación por taxis, sin dificultad, encontrando el intestino en buenas condiciones sin datos de necrosis ni perforación (Fig. 2). Su evolución posoperatoria fue satisfactoria, sin complicaciones, y fue dada de alta a los 5 días.

\section{Discusión}

Las invaginaciones intestinales posoperatorias son extremadamente raras y la mayoría se presentan dentro de los primeros 7 días tras la realización de un

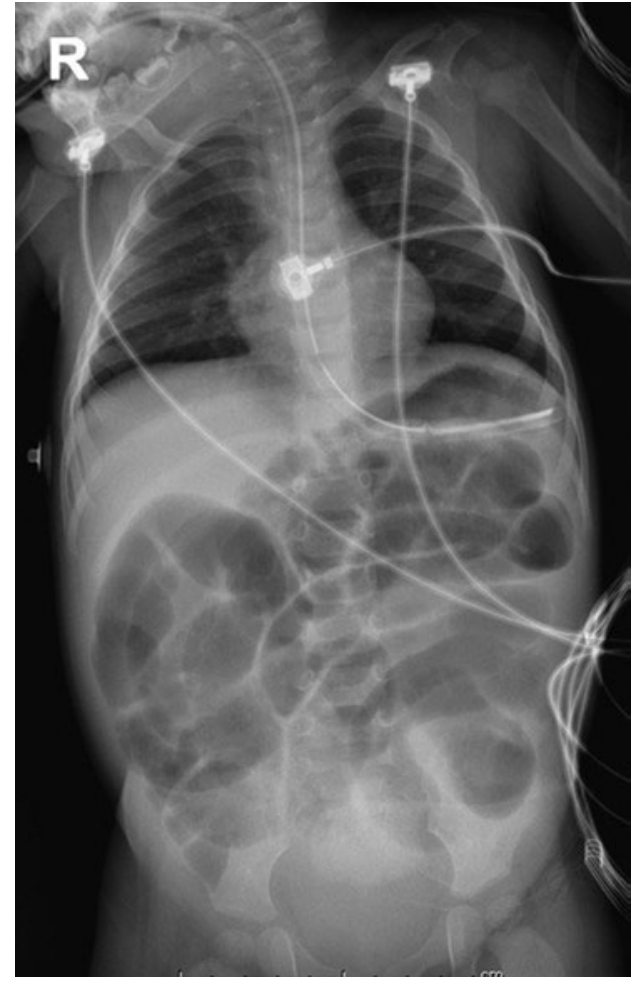

Figura 1. Radiografía simple de abdomen que muestra una dilatación importante de asas de intestino delgado.

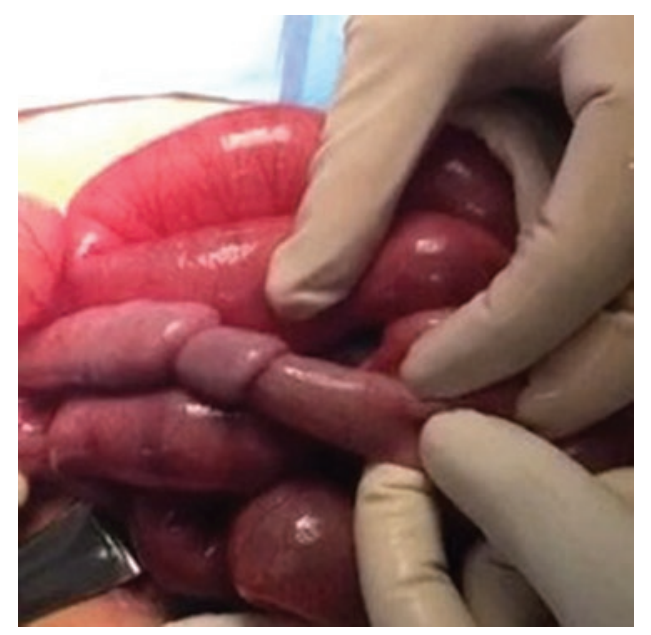

Figura 2. Doble invaginación íleo-ileal a $60 \mathrm{~cm}$ de la válvula ileocecal.

procedimiento quirúrgico. Las cirugías más frecuentemente relacionadas con invaginación intestinal posoperatoria son las operaciones gastrointestinales $(51.2 \%)$, la resección de tumor retroperitoneal (20.5\%), las operaciones que involucran el diafragma (9.4\%), las del sistema urinario (6.3\%), la pancreatectomía $(3.9 \%)$ y otras operaciones no abdominales $(8.7 \%)^{1-6}$.

Las manifestaciones clínicas de esta condición son atípicas, por lo que a menudo recibe atención insuficiente. Una vez que ocurre, es bastante difícil hacer 
un diagnóstico preoperatorio definitivo. Cualquier retraso en el diagnóstico y el tratamiento puede ser potencialmente mortal y tener consecuencias graves, como la necrosis del intestino. Por lo tanto, el diagnóstico temprano y la exploración quirúrgica son esenciales. Los síntomas principales referidos son vómitos biliosos, distensión abdominal y dolor abdominal, así como ausencia de evacuaciones ${ }^{1-6}$.

A diferencia de la invaginación idiopática, la mayoría de las invaginaciones posoperatorias pueden afectar a cualquier grupo de edad; el rango de edad se extiende de 1 semana a 15 años, y la edad promedio es de 5,1 años. Solo tres de los niños tenían menos de 2 años; el resto tenían más de 3 años ${ }^{3,5}$. Casi siempre afecta al intestino delgado. Se refiere que hasta en un $85.6 \%$ de los casos las invaginaciones son íleo-iliales, seguidas de la yeyuno-yeyunal, y son muy raras las íleo-cólicas ${ }^{1,3,5}$.

La mayoría de las invaginaciones se resuelven mediante laparotomía y reducción manual. Solo un pequeño porcentaje (7.4\%) requiere resección y anastomosis $1,2,4,5$.

La etiología de la invaginación posoperatoria es desconocida. Se presume que se debe a múltiples factores que incluyen trauma quirúrgico, edema de íleon, hipoxia, exposición extensa o manipulaciones prolongadas del intestino, estímulos neurogénicos, agentes anestésicos, quimioterapia o radiación, y otros. La mayoría de los autores mencionan una disparidad en la reanudación de la peristalsis intestinal causada por un traumatismo quirúrgico, con posible espasmo local o edema de la pared del intestino delgado como explicación más plausible ${ }^{2,4}$.

En cualquier íleo posoperatorio atípico se recomienda realizar un estudio ecográfico para descartar el diagnóstico de intususcepción posoperatoria, lo cual no se llevó a cabo en nuestro caso ya que no se sospechó la posibilidad de que tuviera una invaginación intestinal. Una vez diagnosticada esta condición, el tratamiento quirúrgico debe realizarse tan pronto como sea posible 2,4 .

La laparotomía con reducción manual es el tratamiento descrito más frecuente. La resección intestinal y la anastomosis rara vez son necesarias ${ }^{2}$.

\section{Conclusiones}

Debe sospecharse una invaginación posoperatoria en pacientes quirúrgicos pediátricos que muestren signos de obstrucción intestinal en el posoperatorio temprano. En cualquier íleo posoperatorio atípico o ante la presencia de datos clínicos de oclusión intestinal se recomienda realizar un estudio ecográfico para descartar el diagnóstico de invaginación posoperatoria. Se recomienda realizar una laparotomía inmediata para minimizar la morbilidad y la mortalidad de esta complicación quirúrgica.

\section{Conflicto de intereses}

Los autores declaran que no existe conflicto de intereses.

\section{Financiamiento}

Los autores declaran que no se obtuvo financiamiento de ninguna organización o institución para la realización de este artículo.

\section{Responsabilidades éticas}

Protección de personas y animales. Los autores declaran que para esta investigación no se han realizado experimentos en seres humanos ni en animales.

Confidencialidad de los datos. Los autores declaran que han seguido los protocolos de su centro de trabajo sobre la publicación de datos de pacientes.

\section{Derecho a la privacidad y consentimiento infor-} mado. Los autores han obtenido el consentimiento informado de los pacientes y/o sujetos referidos en el artículo. Este documento obra en poder del autor de correspondencia.

\section{Bibliografía}

1. Yang G, Wang X, Jiang W, Ma J, Zhao J, Liu W. Postoperative intussusceptions in children and infants: a systematic review. Pediatr Surg Int. 2013;29:1273-9.

2. Van Houwelingen L, Seims A, Ortega-Laureano L, Coleman J, McCarville $M$, Davidoff $A$, et al. Use of ultrasound in diagnosing postoperative small bowel intussusception in pediatric surgical oncology patients: a single-center retrospective review. Pediatr Radiol. 2018;48:204-9.

3. Olcay I, Zorludemir U. Idiopathic postoperative intussusception. Z Kinderchir.1989;44:86-7.

4. Bai $\mathrm{Y}$, Chen $\mathrm{H}$, Wang W. A special type of postoperative intussusception: ileoileal intussusception after surgical reduction of ileocolic intussusception in infants and children. J Pediatr Surg. 2009;44:755-8.

5. West K, Stephens B, Rescorla F, Vane D, Grosfeld J. Postoperative intussusception: experience with 36 cases in children. Surgery. 1988; 104:781-7.

6. Türkyilmaz Z, Sönmez K, Demiroğullari B, Karabulut R, Ozen I, Moralioğlu S, et al. Postoperative intussusception in children. Acta Chir Belg. 2005;105:187-9. 